\title{
Field-induced criticality in a gapped quantum magnet with bond disorder
}

\author{
D. Hüvonen, ${ }^{1}$ S. Zhao, ${ }^{1}$ M. Månsson, ${ }^{1}$ T. Yankova, ${ }^{1,2}$ E. Ressouche,${ }^{3}$ C. Niedermayer, ${ }^{4}$ M. Laver, ${ }^{4,5,6}$ \\ S. N. Gvasaliya, ${ }^{1}$ and A. Zheludev ${ }^{1, *}$ \\ ${ }^{1}$ Neutron Scattering and Magnetism, Laboratory for Solid State Physics, ETH Zurich, Switzerland \\ ${ }^{2}$ Chemistry Dept., Lomonosov Moscow State University, Russia \\ ${ }^{3}$ INAC/SPSMS-MDN, CEA/Grenoble, 17 rue des Martyrs, 38054 Grenoble Cedex 9, France \\ ${ }^{4}$ Laboratory for Neutron Scattering, Paul Scherrer Institute, CH-5232 Villigen, Switzerland \\ ${ }^{5}$ Materials Research Division, Ris DTU, Technical University of Denmark, DK-4000 Roskilde, Denmark \\ ${ }^{6}$ Nano Science Center, Niels Bohr Institute, University of Copenhagen, DK-2100 Copenhagen, Denmark
}

(Received 27 January 2012; published 30 March 2012)

\begin{abstract}
Neutron diffraction and calorimetric measurements are used to study the field-induced quantum phase transition in piperazinium- $\mathrm{Cu}_{2}\left(\mathrm{Cl}_{1-x} \mathrm{Br}_{x}\right)_{6}(x=0,3.5 \%$, and 7.5\%), a prototypical quantum antiferromagnet with random bonds. The critical indexes $\phi$ and $\beta$ are determined. The findings contradict some original predictions for Bose glass based on the assumption $z=d$, but are consistent with recent theoretical results implying $z \leqslant d$. Inelastic neutron experiments reveal that disorder has a profound effect on the lowest-energy magnetic gap excitation in the system.
\end{abstract}

DOI: 10.1103/PhysRevB.85.100410

PACS number(s): 75.10.Jm, 64.70.Tg, 75.40.Cx, 72.15.Rn

The persistent interest in quantum magnets is driven by their use as prototypes for the study of quantum critical phenomena. In this context, much attention has been given to field-induced phase transitions in gapped spin systems with a singlet ground state, the so-called quantum spin liquids. Such transitions are described in terms of a Bose-Einstein condensation (BEC) of magnons. ${ }^{1}$ The specific advantage of magnetic realizations of BEC lies in the relatively easy experimental access to-and control of - the key relevant quantities, notably the effective chemical potential, boson density, and BEC order parameter. In magnetic $\mathrm{BEC}$, the latter corresponds to magnetic field, magnetization, and antiferromagnetic order, respectively.

A particular area of recent interest is BEC in the presence of a spatially random potential. Such disorder leads to a localization of bosons and the appearance of a novel quantum phase, the so-called Bose glass. ${ }^{2-4}$ Most importantly, it changes the universality class of the quantum critical point. While in the disorder-free case it is mean-field (MF) -like with dynamical critical exponent $z=2,{ }^{1}$ very unusual scaling with $z$ equal to dimensionality $(z=d)$ was predicted for disordered systems. ${ }^{3}$ This premise was recently challenged, ${ }^{5,6}$ suggesting that in fact $z \leqslant d .^{7,8}$ Quantum magnetic materials offer a unique opportunity of addressing this important matter of debate experimentally. A random potential for magnons can be created by randomizing the strength of magnetic interactions. This is achieved through a spatially random chemical modification. Indications of the magnetic Bose glass ${ }^{9-11}$ and a modification of the field-induced ordering transition ${ }^{12-14}$ have been reported. However, experiments only added to the confusion regarding critical indexes and scaling. Recent studies of $\mathrm{Tl}_{0.64} \mathrm{~K}_{0.36} \mathrm{CuCl}_{3}$ seemed to validate the $z=d$ hypothesis. ${ }^{12}$ Other experiments, on the organic compound DTN, ${ }^{13}$ yielded quite different critical exponents and were supported by density matrix renormalization group (DMRG) calculations. In the present work, we seek to resolve the issue through neutron and calorimetric studies of the chemically disordered quantum magnets piperazinium- $\mathrm{Cu}_{2}\left(\mathrm{Cl}_{1-x} \mathrm{Br}_{x}\right)_{6}$ (PHCX). We determine the critical exponents $\phi$ and $\beta$, the latter previously unmeasured for this type of transition. We find experimental values that are clearly inconsistent with predictions based on $z=d$.

One rationale for selecting $\mathrm{PHCX}$ as a test material is that the disorder-free Cl-rich $(x=0)$ compound (PHCC) is an exceptionally well-characterized Heisenberg quantum magnet. ${ }^{15-18}$ It features a complex quasi-two-dimensional spin network of $S=1 / 2 \mathrm{Cu}^{2+}$ ions bridged by $\mathrm{Cu}-\mathrm{Cl}-\mathrm{Cl}-$ $\mathrm{Cu}$ superexchange pathways; see Fig. 1 in Ref. 15. The exact Hamiltonian for this system remains unknown with six to eight possible superexchange pathways within the $\mathrm{Cu}_{2} \mathrm{Cl}_{6}$ layers. However, for the physics discussed here, a relevant ramification is the spin liquid ground state and the gapped $(\Delta=1 \mathrm{meV}$ ) magnon spectrum with a bandwidth of about $1.7 \mathrm{meV}$. In magnetic fields exceeding $\mu_{0} H_{c}=7.5 \mathrm{~T}$, PHCC undergoes a quantum phase transition to a state with spontaneous antiferromagnetic (AF) order perpendicular to the direction of applied field. The critical properties of this transition have been investigated using a variety of techniques. ${ }^{17}$ The two critical exponents studied in this work are the order-parameter exponent $\beta$ and the so-called crossover exponent $\phi$. The former defines the field dependence of the AF ordered moment at $T \rightarrow 0$ : $\left|\left\langle\mathbf{S}_{\perp}\right\rangle\right| \sim\left(H-H_{c}\right)^{\beta}$, where $H_{c} \equiv H_{c}(T=0)$. The crossover index $\phi$ defines the phase boundary of the ordered state on the $H-T$ phase diagram: $T_{c}(H) \sim\left(H-H_{c}\right)^{\phi}$ or $T_{c}(H)^{1 / \phi} \sim\left(H-H_{c}\right)$.

$\mathrm{Br}$ substitution in PHCX is expected to locally affect the bond angles in the halogen-mediated superexchange routes, and thereby to realize the random bond model. This mechanism was previously exploited in the study of random-bond systems IPA-CuCl 3 , ${ }^{9,11} \mathrm{Sul}-\mathrm{Cu}_{2} \mathrm{Cl}_{4},{ }^{14}$ and DTN.${ }^{13}$ High-quality PHCX single crystals were grown from solution as described in Ref. 19. Single-crystal x-ray diffraction confirmed that for $\mathrm{Br}$ content $x$ up to at least $10 \%$, the crystal symmetry remains intact, space group $(P \overline{1})$. The lattice parameters are $a=7.9691(5) \AA, b=7.0348(5) \AA, c=6.0836(4) \AA$, $\alpha=111.083(3)^{\circ}, \beta=99.947(3)^{\circ}$, and $\gamma=81.287(4)^{\circ}$ for the pure material with $x=0,{ }^{20}$ and the cell dimensions increase 

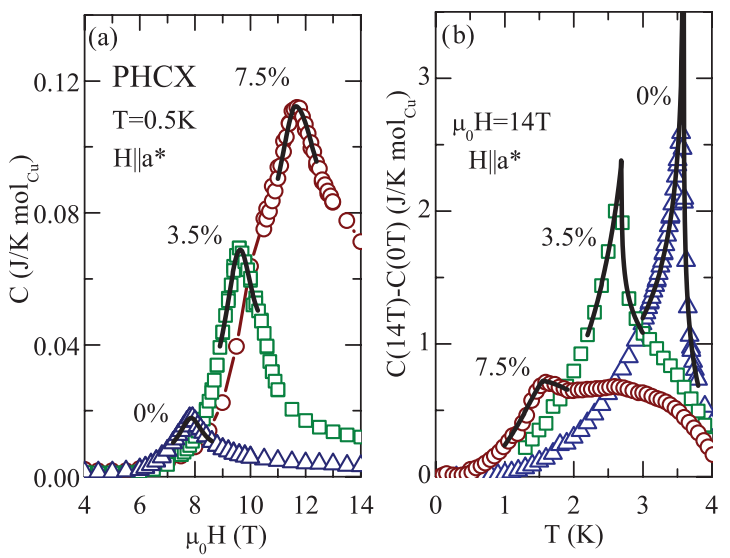

FIG. 1. (Color online) Symbols: Heat capacity of PHCX with varying $\mathrm{Br}$ concentration $x$ measured (a) as a function of magnetic field at $T=0.5 \mathrm{~K}$ and (b) as a function of temperature at $H=14 \mathrm{~T}$. The solid lines are fits to the data as described in the text.

linearly with $x$. Here $x$ stands for nominal $\mathrm{Br}$ concentration in the starting solution. Studies on similar compounds have shown a very good correspondence between nominal and final dopant concentrations. ${ }^{14,19}$ Most of the work reported here was done on perfectly faceted single crystals with $x=3.5 \%$ and $7.5 \%$, where $a=7.9799(2) \AA, b=7.0406(2)$ $\AA, c=6.09090(10) \AA$ and $a=7.9905(3) \AA, b=7.0454(2)$ $\AA, c=6.1000(2) \AA$, respectively, with the triclinic angles indistinguishable from the pure compounds.

The crossover exponent was derived from calorimetric measurements. For $x=0,3.5 \%$, and $7.5 \%$, the data were collected using a $14 \mathrm{~T}$ Quantum Design PPMS with a ${ }^{3} \mathrm{He}-{ }^{4} \mathrm{He}$ dilution insert, both as a function of temperature and magnetic field applied along the $a^{*}$ axis. Typical scans are shown in Figs. 1(a) and 1(b) (symbols). Sharp $\lambda$ anomalies are apparent in all cases and mark the ordering transition. The apparent broadening at low $T$ for the $x=7.5 \%$ sample is of instrumental origin. It is caused by differences in the slope of the phase boundary in conjunction with finite-temperature resolution. In the constant-field scans, the actual transition temperatures $T_{c}$ were determined by fitting the data at each field, in the vicinity of the peaks, to an empirical form of power law with broadening. ${ }^{21} \mathrm{~A}$ similar approach was used to obtain the temperature dependence of the critical field $H_{c}$ from constant-temperature scans. In this case, the fit function was a composite of two half-Lorentzians of nonequal widths and common maxima. The resulting phase boundaries are plotted in Fig. 2 (symbols). Clearly, the introduction of disorder shifts the transition to higher fields and lower temperatures.

Any additional quantitative analysis has to take into account the experimental errors. For consistency, we only used the more comprehensive data set obtained in constant temperature scans (empty symbols in Fig. 2). The error bar on $H_{c}$ was estimated as the standard deviation of this parameter in the least-squares fits described above and is significantly smaller than the symbol size in the plot. The dominant error on $T_{c}$ is of instrumental origin and is due to the use of the temperature relaxation measurement method. It is defined by the magnitude of applied heat pulse (3\% of the starting temperature in our case).
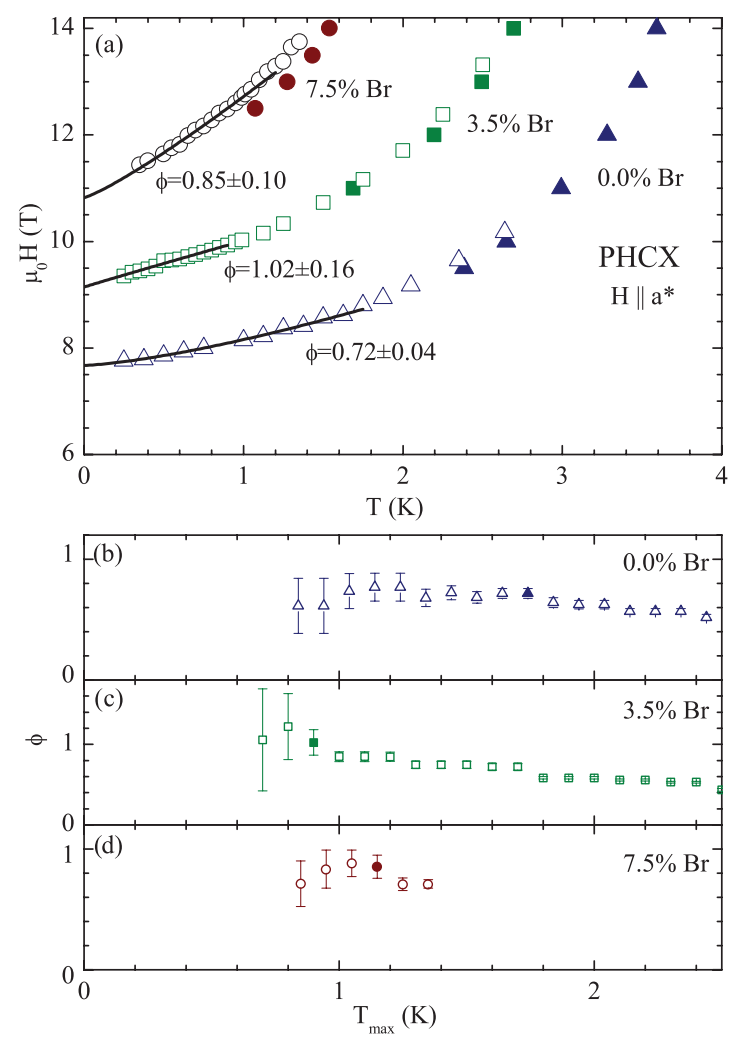

FIG. 2. (Color online) (a) Phase boundaries of an ordered state for $\mathrm{Br}$-free, $3.5 \% \mathrm{Br}$, and 7.5\% $\mathrm{Br}$ substituted PHCX. Open and filled circles are from $C(H)$ and $C(T)$ measurements, respectively. Solid lines are power laws $H-H_{c} \sim T_{c}(H)^{1 / \phi}$ with critical fields $H_{c}$ and crossover exponents $\phi$ derived from the data as described in the text. (b)-(d) Crossover exponent $\phi$ obtained from a least-squares fit as a function of a fitting window given by $T<T_{\max }$. Filled symbols show the value quoted in Table I satisfiying our best-fit criteria described in the text.

The exponent $\phi$ is obtained by a weighted least-squares fit of the power-law function to the experimental field dependence of $T_{c}$ accounting for experimental errors on both. This deceptively simple procedure is actually quite delicate. The fit is performed in a progressively shrinking data range $T<T_{\max } \rightarrow 0$. In Figs. 2(b)-2(d), we show the fitted values of $\phi$ for the three materials plotted against $T_{\max }$. The range used for the final fits is to be selected based on two conflicting requirements. It has to be as narrow as possible in order to access the true critical region. This desire is counteracted by the divergence of confidence intervals at $T_{\max } \rightarrow 0$. As a rule of thumb, one can choose the largest $T_{\max }$ that gives a value of $\phi$ within the confidence intervals obtained using any narrower ranges. Further reducing the fitting range does not produce a statistically significant change in the result.

Returning to our case of PHCC, based on the plots in Fig. 2 and the above-mentioned rule of thumb, for the $x=0,3.5 \%$, and $7.5 \%$ materials we have selected fitting ranges $T_{\max }=$ $1.75,0.9$, and $1.15 \mathrm{~K}$, respectively. The resulting values of $\phi$ and $H_{c}$ are summarized in Table I. Power laws derived from these parameter values are shown as solid lines up to $T=T_{\max }$ in Fig. 2. 
TABLE I. Experimental estimates for the critical field $H_{c}$ in $\mathbf{H} \| \mathbf{a}^{*}$ orientation and the critical exponents $\phi$ (from calorimetric data) and $\beta$ (from neutron diffraction) of the field-induced quantum phase transition in PHCX in comparison with recent measurements on similar systems and theoretical results, the relevance of which is discussed in the text.

\begin{tabular}{lccc}
\hline \hline & $\mu_{0} H_{c}(\mathrm{~T})$ & $\phi$ & $\beta$ \\
\hline PHCX 0.0\% Br & $7.67 \pm 0.02$ & $0.72 \pm 0.04$ & $0.40 \pm 0.01($ Ref. 17) \\
PHCX 3.5\% Br & $9.14 \pm 0.08$ & $1.02 \pm 0.16$ & $0.45 \pm 0.05$ \\
PHCX 7.5\% Br & $10.82 \pm 0.14$ & $0.85 \pm 0.10$ & $0.42 \pm 0.02$ \\
DTN 0.8\% Br (Ref. 13) & $1.07 \pm 0.01$ & $1.1 \pm 0.2$ & 1.7 \\
Tl ${ }_{0.64} \mathrm{~K}_{0.36} \mathrm{CuCl}_{3}$ (Ref. 12) & $2.7 \pm 0.6$ & $\geqslant 2$ & 0.61 \\
$z=d$ scaling (Ref. 3) & & 1.54 & 0.5 \\
QMC (Ref. 7) & & $1.06 \pm 0.09$ & 0.667 \\
QMC (Ref. 13) & & \\
BEC (MF) & &
\end{tabular}

The behavior of the magnetic BEC order parameter was studied in neutron diffraction experiments. For the $x=3.5 \%$ and $7.5 \%$ samples, these were carried out at the CRG-CEA D23 diffractometer at ILL and the RITA-II three-axis spectrometer at PSI, respectively. In the two experiments, we used a $\mathrm{Cu}$ monochromator to produce neutrons of incident energies $50 \mathrm{meV}$ at ILL and pyrolitic graphite for $4.6 \mathrm{meV}$ neutrons with a Be filter in front of the analyzer at PSI. The sample environment was in all cases a split coil cryomagnet with a ${ }^{3} \mathrm{He}-{ }^{4} \mathrm{He}$ dilution cryostat. The magnetic field was applied along $\mathbf{a}^{*}$ in the first and along the $\mathbf{b}$ crystallographic axis in the second experiment. As previously reported for PHCC, ${ }^{17}$ longrange order in the BEC phase is marked by the appearance of new magnetic Bragg reflection half-integer reciprocal-lattice points $(h+1 / 2,0, l+1 / 2)$. The inset in Fig. 3(b) shows typical scans across the $(0.5,0,-0.5)$ position measured in the $x=7.5 \%$ sample at $T=0.5 \mathrm{~K}$ in the BEC phase and the background collected at $T=2 \mathrm{~K}$ in the paramagnetic state. To within experimental error, all magnetic reflections are resolution-limited.

The critical index $\beta$ was determined from measurements of the $(0.5,0,-0.5)$ Bragg peak intensity in zero-field-cooled (ZFC) samples. These data were taken upon increasing the applied field at $T=160 \mathrm{mK}$ and are plotted in Fig. 3(a). $\beta$ was then determined in power-law fits, assuming that peak intensity scales as the square of the order parameter. As for $\phi$, the fits for $\beta$ were performed for a series of shrinking fitting intervals, of magnetic field $\delta H=H-H_{c}$ in this case. The fit results and standard deviation confidence intervals are plotted against $\delta H$ in Figs. 3(c) and 3(d). Following the reasoning described earlier, in our final analysis we have selected $\mu_{0} \delta H=1.86$ and $2.15 \mathrm{~T}$ for $x=3.5 \%$ and $7.5 \%$, respectively. The resulting exponents are summarized in Table I. In order to properly characterize the quantum critical point, one has to know $\beta$ in the limit $T \rightarrow 0$. Previous studies of PHCC suggest that $\beta$ remains practically constant for $T<0.3 \mathrm{~K} .{ }^{17}$ We have additionally verified this behavior for our $3.5 \%$ PHCX sample. Thus, the $160 \mathrm{mK}$ measurements are good estimates of the zero-temperature values. Comparing our results with those reported in Ref. 17, we conclude that disorder does not significantly change the criticality of the order parameter.
This is not to say that the effect of disorder is negligible. Inelastic neutron scattering experiments performed at zero field on the TASP three-axis spectrometer ${ }^{22}$ at PSI using $3.5 \mathrm{meV}$ incident-energy neutrons and a Be filter reveal that while the magnons are sharp in $\mathrm{PHCC}^{17}$ they acquire a
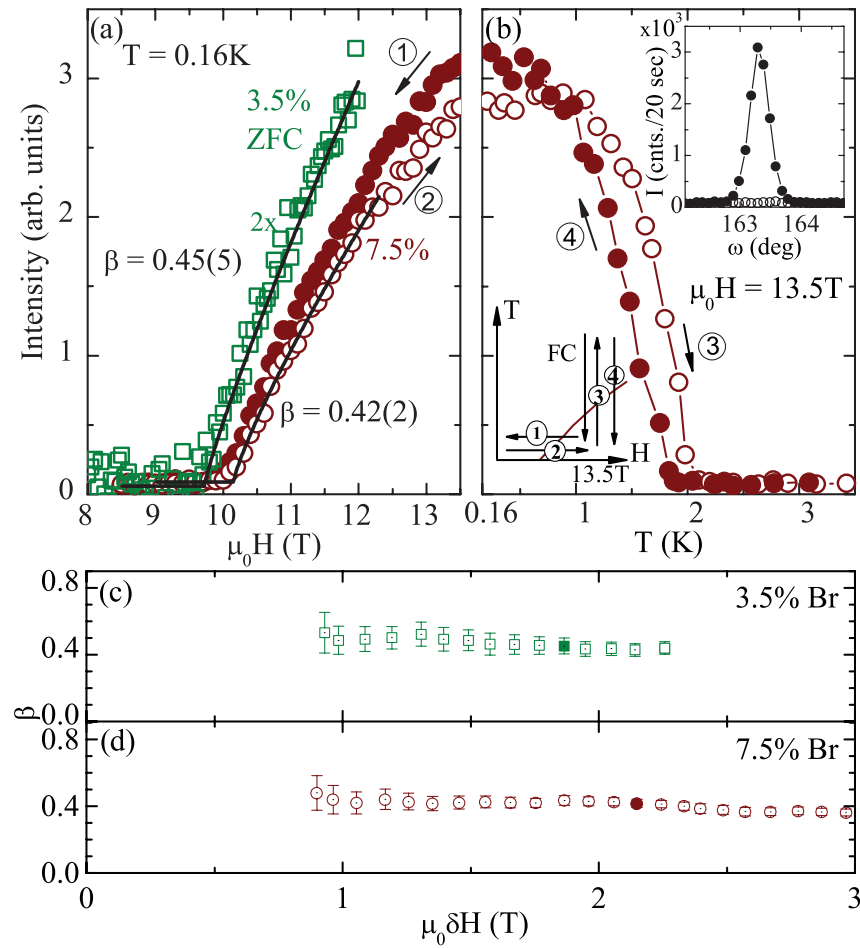

FIG. 3. (Color online) Upper panels: Neutron diffraction intensity of the $\left(\frac{1}{2} 0 \frac{\overline{1}}{2}\right)$ peak measured in PHCX, (a) as a function of magnetic field at $T=160 \mathrm{mK}$, (b) as a function of temperature. Open and solid symbols correspond to zero field cooling and field cooling, respectively. Squares (circles) correspond to $x=3.5 \%(7.5 \%) \mathrm{Br}$ content. Lower left corner: the trajectory in $(H, T)$ space for the measurements on the $x=7.5 \%$ material. Inset: rocking curve measured at $T=0.5 \mathrm{~K}$ (filled) and $T=2 \mathrm{~K}$ (empty circles) in that sample. Lower panels: order-parameter exponents as obtained by shrinking the fitting interval analysis of the $3.5 \%$ (c) and $7.5 \% \mathrm{Br}$ (d) sample data. Filled symbols show the best-fit values of $\beta$. 


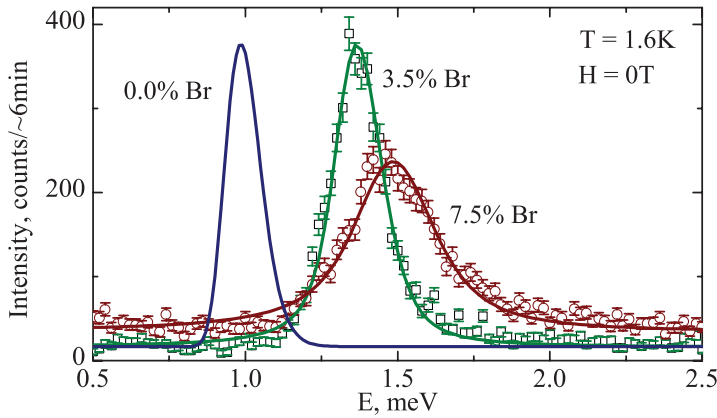

FIG. 4. (Color online) Inelastic neutron scattering intensity at $(0.5,0.5,-0.5)$ in the energy transfer range from 0.5 to $2.5 \mathrm{meV}$ for $x=3.5 \%$ (green squares) and $x=7.5 \% \mathrm{Br}$ (red circles) content samples. Corresponding solid lines are the fitted Lorentzian energy profiles convoluted with the four-dimensional instrumental resolution function. For $0.0 \% \mathrm{Br}$ sample the resolution convoluted simulation is shown for comparison using parameters from Ref. 15

considerable intrinsic width in disordered samples. Typical constant-q scans at the AF zone center $(0.5,0.5,-0.5)$ in the $x=3.5 \%$ and $7.5 \%$ samples are shown in Fig. 4 by the open symbols. The solid lines are fits of Lorentzian energy profiles convoluted with the four-dimensional instrumental resolution function. ${ }^{23}$ For $x=0 \%$, the line is a simulation assuming that peak shape is determined solely by resolution. The observed increase of gap energy with $\mathrm{Br}$ substitution is fully consistent with the observed increase of critical field and may be attributed to a change in the disorder-averaged exchange constants. However, the large intrinsic energy width $\Gamma=0.1$ and $0.25 \mathrm{meV}$ for the $x=3.5 \%$ and $7.5 \%$ samples, respectively, is entirely a disorder-induced effect. ${ }^{24}$

Our present diffraction experiments show that disorder also affects the behavior of the order parameter deep within the high-field phase. In the $7.5 \%$ material, the intensity of the magnetic reflection exhibits a history dependence. This is illustrated in Figs. 3(a) and 3(b). Here we show data collected along a trajectory illustrated in the inset. No such behavior was reported for the disorder-free material, but a similar and much stronger history effect was previously studied in a related system, IPA- $\left(\mathrm{Cu}_{1-x} \mathrm{Br}_{x}\right) \mathrm{Cl}_{3} .{ }^{11} \mathrm{~A}$ related phase-transition scenario that could lead to hysteretic behavior was discussed in Ref. 25.

Theoretical and numerical predictions for the two measured critical exponents are shown in Table I for a direct comparison with our experiments. In the absence of disorder, one expects MF values ${ }^{17} \beta=1 / 2$ and $\phi=2 / 3$, in reasonable agreement with the data for PHCC. Assuming $z=d$, the disordered case gives much larger value for the crossover exponent $\phi \geqslant 2{ }^{26}$ This prediction is clearly inconsistent with our experiments. More recent numerical work ${ }^{7,8,13}$ suggested smaller values of the exponent. Those from Refs. 7 and 8 are less relevant to three-dimensional ordering in PHCX, as they are obtained for a two-dimensional model. On the other hand, the QMC calculations of Ref. 13 should be directly comparable to our data. The corresponding estimates of $\phi$ are in rather good agreement with our experiments, as well as with those on the Br-substituted DTN compound. ${ }^{13}$ A relevant theoretical study of $\beta$ is presently lacking. The claimed observation ${ }^{12}$ of values consistent with $z=d$ can be due to overinterpretation of a limited dataset. Evidence is mounting for the breakdown of the $z=d$ hypothesis in the Bose glass to BEC quantum phase transition.

This work is partially supported by the Swiss National Fund under project 2-77060-11 and through Project 6 of MANEP. M.L. acknowledges support from DanScat. We thank Dr. V. Glazkov for his involvement in the early stages of this project. *http://www.neutron.ethz.ch/

${ }^{1}$ T. Giamarchi, C. Ruegg, and O. Tchernyshev, Nat. Phys. 4, 198 (2008).

${ }^{2}$ T. Giamarchi and H. J. Schulz, Europhys. Lett. 3, 1287 (1987).

${ }^{3}$ M. P. A. Fisher, P. B. Weichman, G. Grinstein, and D. S. Fisher, Phys. Rev. B 40, 546 (1989).

${ }^{4}$ O. Nohadani, S. Wessel, and S. Haas, Phys. Rev. Lett. 95, 227201 (2005).

${ }^{5}$ P. B. Weichman and R. Mukhopadhyay, Phys. Rev. Lett. 98, 245701 (2007).

${ }^{6}$ P. B. Weichman and R. Mukhopadhyay, Phys. Rev. B 77, 214516 (2008).

${ }^{7}$ A. Priyadarshee, S. Chandrasekharan, J.-W. Lee, and H. U. Baranger, Phys. Rev. Lett. 97, 115703 (2006).

${ }^{8}$ H. Meier and M. Wallin, Phys. Rev. Lett. 108, 055701 (2012).

${ }^{9}$ H. Manaka, A. V. Kolomiets, and T. Goto, Phys. Rev. Lett. 101, 077204 (2008).

${ }^{10}$ H. Manaka, H. A. Katori, O. V. Kolomiets, and T. Goto, Phys. Rev. B 79, 092401 (2009).

${ }^{11}$ T. Hong, A. Zheludev, H. Manaka, and L.-P. Regnault, Phys. Rev. B 81, 060410 (2010).
${ }^{12}$ F. Yamada, H. Tanaka, T. Ono, and H. Nojiri, Phys. Rev. B 83, 020409 (2011)

${ }^{13}$ R. Yu, L. Yin, N. S. Sullivan, J. S. Xia, C. Huan, A. Paduan-Filho, N. F. Oliveira Jr., S. Haas, A. Steppke, C. F. Miclea, F. Weickert, R. Movshovich, E.-D. Mun, V. S. Zapf, and T. Roscilde, e-print arXiv:1109.4403v2.

${ }^{14}$ E. Wulf, S. Mühlbauer, T. Yankova, and A. Zheludev, Phys. Rev. B 84, 174414 (2011).

${ }^{15}$ M. B. Stone, I. Zaliznyak, D. H. Reich, and C. Broholm, Phys. Rev. B 64, 144405 (2001).

${ }^{16}$ M. B. Stone, C. Broholm, D. H. Reich, O. Tchernyshyov, P. Vorderwisch, and N. Harrison, Phys. Rev. Lett. 96, 257203 (2006).

${ }^{17}$ M. B. Stone, C. Broholm, D. H. Reich, P. Schiffer, O. Tchernyshyov, P. Vorderwisch, and N. Harrison, New J. Phys. 9, 31 (2007).

${ }^{18}$ M. B. Stone, I. A. Zaliznyak, T. Hong, C. L. Broholm, and D. H. Reich, Nature (London) 440, 187 (2006).

${ }^{19}$ T. Yankova, D. Hüvonen, S. Muehlbauer, D. Schmidiger, E. Wulf, S. Zhao, A. Zheludev, T. Hong, V. O. Garlea, R. Custelcean, and G. Ehlers, e-print arXiv:1110.6375v1.

${ }^{20}$ G. Marcotrigiano, L. Menabue, and G. C. Pellacani, Inorg. Chem. 15, 2333 (1976). 
${ }^{21} C(T)=b-a_{l, r} \gamma^{-2}-a_{l, r}\left(T-T_{c}^{\alpha_{l, r}}+\gamma^{2}\right)^{-1}$, where $\gamma$ is a broadening parameter, $b$ is background, and $a_{l, r}$ and $\alpha_{l, r}$ are scaling constants and scaling exponents on the left and right side of $T_{c}$, respectively.

${ }^{22}$ F. Semadeni, B. Roessli, and P. Boni, Physica B 297, 152 (2001).

${ }^{23}$ M. Popovici, Acta Cryst. A31, 507 (1975) .

${ }^{24} \mathrm{~A}$ full account of the inelastic neutron scattering experiments is beyond the scope of the present paper, which is focused on the field-induced transitions. Such an account will be given elsewhere.
${ }^{25}$ R. Yu, S. Haas, and T. Roscilde, Europhys. Lett. 89, 10009 (2010).

${ }^{26}$ Lower limit on $\phi$ follows from Eqs. 5.8 and 5.10 in Ref. 3 as follows: $T_{c} \propto\left[\rho_{s}(0)\right]^{x}$ and $\rho_{s}(0) \propto\left(\rho-\rho_{c}\right)^{\zeta}$ with $x=z /(d+z-2)$ and $\zeta=v(d+z-2)$. Since magnetization and boson density are proportional, $\left(\rho-\rho_{c}\right) \propto\left(H-H_{c}\right)$, one obtains $T_{c} \propto\left(H-H_{c}\right)^{x \zeta}=$ $\left(H-H_{c}\right)^{z v}$. Since $v \geqslant 2 / d$ (Eq. 3.24 in Ref. 3) for all dimensions in the presence of disorder and $z=d$, we obtain dimension invariant $\phi \geqslant 2$. 